\title{
Puerto Rico's population before and after Hurricane Maria
}

\author{
Fernando I. Rivera ${ }^{1}$ (D) \\ Published online: 29 July 2020 \\ (C) Springer Nature B.V. 2020
}

In the last 20 years, the island of Puerto Rico has undergone a series of environmental, social, and economic crises. While the devastation caused by Hurricane Maria in 2017 brought nationwide and international attention to the conditions in Puerto Rico, the island has had a history of exposure to several environmental hazards and risks including tsunamis, floods, tropical storms, coral reef deterioration, severe draught, and coastal erosion. All indicators of the impact of climate change on the island's ecological infrastructure. In addition to these hazards and risks, Puerto Rico's financial crisis dating back to the early 2000s has reduced the capacity to maintain critical energy, health care, transportation, and communications structures. This incapacity magnified the impact of Hurricane Maria resulting in $100 \%$ loss of electricity and communication during the critical first days after the hurricane.

Years of economic struggles have had serious socioeconomic ramifications that have fueled extensive out-migration to the continental USA (Mora et al. 2017). The population of Puerto Rico has decreased from 3,725,789 in 2010 to 3,193,694 in 2019, close to a half a million-population loss in less than 10 years (U.S. Census 2020). Hurricane Maria also exposed the scarcity of population data, issues in collecting new data, and to the value of existing administrative data and vital records. As the authors in this issue point out, Puerto Rico's demographic data were limited in demonstrating the effects of Hurricane Maria's long-term decline in population trough either mortality or migration. Indeed, during the initial months after the Hurricane, it became increasingly difficult to estimate the number of people that left the island. It also became increasingly difficult to estimate the casualties associated with the hurricane. Estimates ranged from an initial official death toll of 64 that was later refuted by a public health study which estimated the death toll close to 4000 deaths (Kishore et al. 2018). Subsequently, a study commissioned by the government of Puerto Rico put the estimated death toll at 2975 deaths (Santos-Burgoa et al. 2018).

Fernando I. Rivera

Fernando.Rivera@ucf.edu

1 Department of Sociology and Puerto Rico Research Hub, University of Central Florida, Orlando, FL, USA 
This special issue of Population and Environment puts together a collection of articles that assess the population of Puerto Rico before and after Hurricane Maria. My involvement with this issue came as a suggestion of Population and Environment's Editor-in-Chief, Elizabeth Fussell after a conversation with scholars presenting and attending a session titled "In the Wake of Hurricane Maria: Sociological Perspectives on Puerto Rico" during the 2018 Americans Sociological Society meetings held in Philadelphia, PA (USA). The session presentations revealed knowledge deficits on post-Hurricane Maria's Puerto Rican population movement, deaths, urban development, environmental justice, poverty, work, and welfare.

The issue includes a paper by José Caraballo-Cueto that provides a review of current population databases on Puerto Rico, including the Decennial Population Census, the Puerto Rico Community Service, the Population Estimates Program, the Bureau of Transportation Statistics, the Behavioral Risk Factor Surveillance System, and the Puerto Rico Labor Survey. The author notes the strengths and limitations of each data source and brings attention to the importance of the local context when analyzing these data.

Papers by Jack DeWaard, Janna E. Johnson, and Stephan D. Whitaker; and Yago Martín, Susan L. Cutter, Zhenlong Li, Christopher T. Emrich, and Jerry T. Mitchell analyzed population movement to and from Puerto Rico after Hurricane Maria. Each paper utilized different data sources to analyzed population movement. For instance, the paper by DeWaard et al. utilized data from the Federal Reserve Bank of New York/ Equifax Consumer Credit Panel to analyze the out and return migration from/to Puerto Rico. The authors discuss the feasibility of utilizing consumer credit data for migration analyses. It also found differences in migration for those in vulnerable places, particularly with respect to water and substandard housing.

Martín et al. utilized Twitter data to track population movement after Hurricane Maria. They found an $8.3 \%$ off-island displacement and relocation destinations after the hurricane to Florida, New York, Texas, and Massachusetts. The paper provides evidence of the usefulness of geotagged Twitter data to complement traditional population movement approaches as it provides rapid data availability and reliability.

The paper by Santos-Lozada et al. provides a longitudinal view of population change in Puerto Rico. US Bureau of Transportation Statistics data were used to investigate net migration flows for Puerto Rico. The analyses provide evidence that, while Puerto Rico experienced an out-migration spike after Hurricane Maria, declining economic health continues to be the primary factor behind Puerto Rico's population decline.

Another set of papers examined the health impacts of Hurricane Maria in relation to excess deaths and the risk of adverse birth outcomes. Michael Spagat and Stijn van Weezel utilized data from the Puerto Rico Vital Statistics System to examine excess deaths after the hurricane. The authors employed a Bayesian linear regression to predict monthly death counts for 2017. The authors discuss the discrepancy of their estimate in comparisons with other studies that have investigated and found higher excess death estimates. The authors suggest that the death excess death concept is not individually, but statistically based and therefore the use of Bayesian regressions and placebo tests are useful techniques to analyze the impact of violent events, such as a hurricane, in future excess death estimations. The findings also showed that Puerto Rico experienced a mortality spike that can be attributed to the impact of Hurricane Maria. 
In their paper, Watkins et al. examined hurricane-related experiences and exposure to environmental hazards on a cohort of participants in a pre-hurricane study investigating prenatal environmental exposures and preterm birth outcomes. They found that pregnant women were vulnerable to increased environmental exposures, in this case higher concentrations of compounds of plasticizers found in PVC plastics and food packaging. They also found that women in the cohort lacked prenatal care which elevated their risk for adverse birth outcomes.

Finally, Yerís H. Mayol-García examined how pre-hurricane Maria poverty status was associated with Puerto Rican children's family migration experiences and living arrangements utilizing data from the American Community Survey and the Puerto Rico Community Survey from 2012 to 2016. She found significant differences in poverty status by migration to the continental USA and family living arrangements.

Overall, this special issue provides useful research approaches and data to study and provide answers to important issues experienced by the Puerto Rican population before and after Hurricane Maria. This demographic knowledge is of vital importance to examine the effects of emergencies and natural hazards, particularly as Puerto Rico continues to recover from Hurricane Maria while experiencing seismic movements and the effects of the COVID-19 pandemic.

In closing, I want to express my deepest gratitude to all the authors for their contributions, the useful comments from reviewers, and to Elizabeth Fussell for initiating and supporting the production of the special issue.

\section{References}

Kishore, N., Marqués, D., Mahmud, A., Kiang, M. V., Rodriguez, I., Fuller, A., et al. (2018). Mortality in Puerto Rico after Hurricane Maria. The New England Journal of Medicine, 379(2), 162-170.

Mora, M. T., Dávila, A., \& Rodríguez, H. (2017). Population, migration, and socioeconomic outcomes among island and mainland Puerto Ricans: La Crisis Boricua. Lexington Books.

Santos-Burgoa, C., Sandberg, J., Suárez, E., Goldman-Hawes, A., Zeger, S., Garcia-Meza, A., et al. (2018). Differential and persistent risk of excess mortality from Hurricane Maria in Puerto Rico: a time-series analysis. The Lancet Planetary Health, 2(11), e478-e488.

United States Census Bureau (2020) American fact finder. https://www.census.gov/quickfacts/PR. Accessed 15 June 2020.

Publisher's note Springer Nature remains neutral with regard to jurisdictional claims in published maps and institutional affiliations. 